\title{
Role of sonography done by clinicians/surgery residents in the diagnosis and management of clinically palpable breast lumps.
}

Thapa AS, Ghimire R, Joshi R, Dongol UMS, Singh DR

Department of Surgery, Kathmandu Medical College Teaching Hospital, Sinamangal,

Kathmandu

Correspondence : Anurag Singh Thapa, Resident, Department of Surgery,

Kathmandu Medical College Teaching Hospital.

Email: thapaanurag@gmail.com

\begin{abstract}
Introduction and Objective: The aim of the study is to correlate the sonographic findings of breast ultrasound done by clinicians/surgery residents with radiologists and also to correlate the sonographic findings of breast ultrasound with cytopathological or histopathological findings.
\end{abstract}

Materials and Methods: A prospective observational study conducted at KMCTH from March 2014 to March 2015. All consecutive patients presenting with clinically palpable breast lumps were included in the study. A sample size of 30 patients was taken. All patients with clinically palpable solid breast lumps were subjected to ultrasound by a clinician/surgical resident. Ethical clearance was taken from the institutional review board and informed consent was taken from the individual patients.

Results: We found that the sonography done by clinicians/surgery residents had $100 \%$ sensitivity, $84.6 \%$ specificity and PPV of 50\%. There was significant correlation between the findings of the surgery residents and radiologists and the cytopathology/histopathology as well.

Conclusion: Breast Sonography performed by clinicians/surgery residents is comparable to radiologists in the diagnosis of clinically palpable breast lumps. It has good correlation with cytopathological and histopathological findings.

Key Words: Sonography, Clinicians, Residents, correlations. 